\title{
MAINTAINING DOMAIN SPECIFIC SIMULATION MODELLING FRAMEWORKS - A CASE STUDY ON MODELLING HYPER ACUTE STROKE PATHWAYS
}

\author{
Dr. Durk-Jouke van der Zee \\ Friso Postema \\ University of Groningen \\ P.O. Box 800, $9700 \mathrm{AV}$, Groningen \\ THE NETHERLANDS \\ d.j.van.der.zee@rug.nl, \\ fh.postema@gmail.com
}

\author{
Dr. Thomas Monks
}

University of Exeter Medical School

South Cloisters, St. Luke's Campus, Heavitree

Road, Exeter EX1 1TE

t.w.m.monks@exeter.ac.uk

\author{
Willemijn J. Maas \\ Dr. Maarten M.H. Lahr \\ Dr. Maarten Uyttenboogaart, \\ Prof. dr. Erik Buskens \\ University Medical Center Groningen \\ P.O. Box 30001, 9700 RB, Groningen \\ THE NETHERLANDS \\ w.j.maas@umcg.nl \\ m.m.h.lahr@umcg.nl, \\ m.uyttenboogaart@umcg.nl, \\ e.buskens@umcg.nl
}

\begin{abstract}
Conceptual simulation models capture system essentials in terms of modelling objectives, model inputs, outputs and content. Their impact on simulation study success is undenied. Guidance towards high quality conceptual models is relevant, as modelling is not easy. Modelling frameworks offer guidance by specifying what to model, by identifying modelling activities, and offering good practices and methods in doing so. In this article we explore the needs for maintenance of modelling frameworks and good policies for doing so, starting from a case study. We show how an existing modelling framework addressing hyper acute stroke pathways is extended to meet system requirements set by a new stroke treatment. We clarify how extensions imply significant efforts that may legitimate the definition of maintenance policies, clarifying what, when and how to maintain a framework. In turn, maintenance effectiveness and efficiency may rely on modelling framework setup, being transparent from a maintenance point of view.
\end{abstract}

Keywords: Conceptual modelling, Modelling Frameworks, Hyper Acute Stroke Pathway

\section{INTRODUCTION}

Conceptual modelling (CM) for simulation, boils down to a process of abstraction in which essential elements of a real or would be system are captured in terms of modelling objectives, model inputs, outputs and content (Robinson, 2008b). Essentially, the conceptual model serves as a linking pin between the initial problem situation and the setup of a coded model and its intended use. Clearly, quality of the conceptual model highly impacts on success of a simulation study.

CM is certainly not easy (Law, 1991). It requires bringing together domain specific knowledge and insights, and disciplinary knowledge, especially operations research, statistics, engineering, and computer science. Furthermore, modelling activities are subject to the specifics of the business 


\section{Van der Zee et al.}

context (budgetary constraints, resource availability, time frame etc.) and (possibly conflicting) stakeholder interests. Clearly, this puts high demands on the analyst's skills. At the same time, it clarifies the need for guidance in doing so.

Robinson (2008a) distinguishes three basic approaches on simulation model development: principles of modelling, methods of simplification, and modelling frameworks. Principles of modelling refer to the general case of simulation modelling. Important examples concern, the need for model simplicity, the advocated policy of incremental modelling, and the good use of metaphors, analogies, and similarities in model creation. Methods of simplification focus on the possibility of reducing model scope and/or its level of detail in order to enhance its feasibility and/or utility, while safeguarding its validity (Van der Zee, 2019). Modelling Frameworks go beyond aforementioned approaches by specifying what to model by providing a procedural approach for detailing a model in terms of its elements, their attributes, and their relationships.

In recent years, several modelling frameworks have been developed. The main differences among modelling frameworks concern their intended field of application, scope and process support. Modelling frameworks tend to address rather broad classes of systems, like operations systems (Robinson 2008b), supply chains (Van der Zee and Van der Vorst, 2005), health systems (Kotiadis et al., 2014), the military (Pace, 1999; Pace, 2000) or the general case, i.e., discrete event dynamic systems (Arbez and Birta, 2010). Furthermore, differences among frameworks are found concerning their scope. Whereas some frameworks focus on capturing just model content (Arbez and Birta, 2010), others consider a wider angle by including an exploration of the problem context, project and modelling objectives, and/or the experimental frame, i.e., model inputs and outputs (Kotiadis, 2007; Robinson 2008b). For overviews of modelling frameworks, see Robinson (2008a, 2019), Karagoz and Demirors (2011), Van der Zee et al. (2011) and Furian et al. (2015).

In recent work we considered the development of domain specific modelling frameworks. They offer refined support for the analyst as a net effect of bringing in domain specific knowledge that is helpful in specifying model inputs, outputs and content (Monks et al., 2017). We found how the development of domain specific modelling frameworks could be legitimated vs. more general modelling frameworks by considering (i) problem complexity - being such that it requires joint effort from both experts in the domain of interest and modelling in order to tackle it, (ii) importance of the problem - being of high value to either industry or society or both, and (iii) future demand and uptake of the framework is sufficient to warrant the effort to develop it. As we felt that aforementioned requirements were met for modelling hyper acute stroke systems (HASPs), we developed a domain specific modelling framework targeting respective systems. However, a newly available stroke treatment and its implications for stroke system setup, suggest a need for maintenance, i.e., extensions, of the modelling framework to capture new system elements and their workings. Motivated by this case example, in this paper, we explore the needs for modelling framework maintenance, and good policies in doing so. To do so we rely on first experiences in extending the initial domain specific modelling framework for hyper acute stroke systems by including domain specific knowledge on the new treatment, and its implication for stroke care organization (Postema, 2019). Insights obtained are assumed to be relevant for the setup, development, uptake and use of modelling frameworks.

This paper is structured as follows. In Section 2 we characterize the hyper acute stroke pathway, and the way the new treatment impacts its organization. Next, in Section 3 the updated modelling framework is discussed. In Section 4 we characterize extensions of the new framework relative to the existing framework, and consider their implications in terms of the need for maintenance and good maintenance policies. Finally, in Section 5, we summarize main conclusions.

\section{ORGANIZING THE HYPER ACUTE STROKE PATHWAY - NEW TREATMENTS}

Stroke can be categorized in two subtypes: ischemic and hemorrhagic stroke, respectively $85 \%$ and $15 \%$ of the patient population. Ischemic strokes occur when a cerebral artery is occluded due to a clot and disrupts blood circulation to the brain, whereas hemorrhagic strokes are usually caused by a rupture of a vessel. This paper focuses on ischemic stroke.

Two main reperfusion treatments are available for ischemic strokes, i.e., intravenous thrombolysis (IVT) and intra-arterial thrombectomy (IAT). Efficacy of both treatments is highly time dependent, as 
Van der Zee et al.
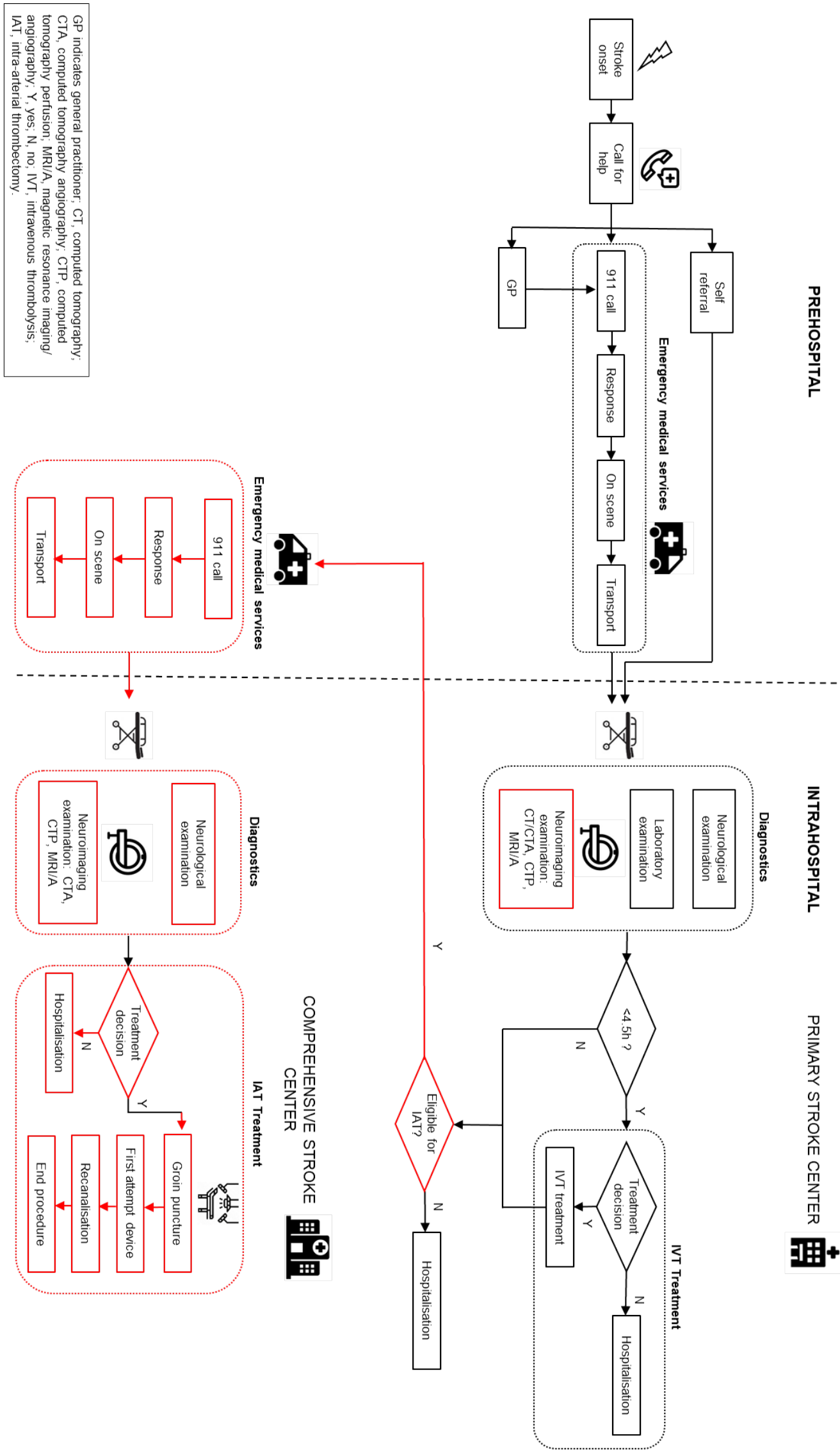

Figure 1 Hyper Acute Stroke Pathway - Drip \& Ship model, i.e., IVT treatment at Primary Stroke Center, follow-up IAT treatment at Comprehensive Stroke Center 


\section{Van der Zee et al.}

clarified by their respective windows of opportunity, i.e., 4.5 and 6 hours after onset (Emberson et al., 2014; Saver et al., 2016). Essentially, IVT leads to recanalization by dissolving the clot, whereas IAT attempts to remove the clot with mechanical devices. IAT is only applicable for stroke patients due to a large vessel occlusion (LVO), concerning about 7\% of the stroke population (Chia et al., 2016). Whereas IVT is provided in many community hospitals acting as Primary Stroke Centers (PSCs), IAT is offered by a limited number of hospitals acting as Comprehensive Stroke Centers (CSCs). Decisions to restrict availability of IAT to designated centers are motivated by the relatively low number of stroke patients facing LVO, and high demands set on staff expertise and availability of specific resources. Current guidelines advocate that LVO patients are treated with IVT (in case of no contraindication for IVT) before receiving IAT treatment.

Whereas IVT is a well-standardised treatment that made an entry in 1995 (The National Institute of Neurological Disorders and Stroke rt-PA Stroke Study Group, 1995), IAT only emerged in recent years (Berkhemer et al., 2014). The availability of IAT and its proven efficacy make adjustments of the organization of the HASP a relevant issue. Two dominant organisation models emerged for serving LVO patients: the "Drip \& Ship" model (DS) and the Mothership model (MS). Figure 1 characterizes the DS model. It clarifies how LVO patients may first be served by a PSC for IVT, and next be transported to the CSC for IAT treatment. Alternatively, those patients in proximity of a CSC may receive both IVT and IAT treatment at the same hospital, i.e., CSC, according to the so-called MS model. Both models imply an extension of the current IVT-based model. Changes implied relative to the IVT-based organisation model, concerning patient routing, new activities, and associated staff and resources are marked red in Figure 1.

Recently, many suggestions have been made to improve organisation models for IAT service, seeking to reduce onset to treatment time. Usually, time of treatment is related to groin puncture, see Figure 1. Suggested improvements of dominant models include, among others, expediting intrahospital workflow, increasing EMS transportation speed by using helicopters, enabling on-call IAT services at PSCs by transporting doctors from a CSC to a PSC or pre-hospital diagnosis seeking to identify LVO patients in an early phase, allowing them to be routed to the CSC directly. Initial findings indicate how the success of proposed interventions is strongly dependent on regional characteristics like current stroke system set-up and resources, medical guidelines, and geography (Ciccone et al., 2019). Unfortunately, a one-fits-all solution does not exist. Hence, optimizing IAT services requires dedicated decision support on a regional scale. Past research has shown how simulation may be a well-qualified means for doing so (Monks et al. 2017).

\section{TOWARDS AN UPDATED SIMULATION MODELLING FRAMEWORK FOR COMBINED IVT-IAT TREATMENT}

This section discusses extensions of the modelling framework proposed by Monks et al. (2017) that is meant to support simulation conceptual modelling of stroke systems for IVT treatment only. Main focus will be on the approach taken for maintaining the framework, the nature of extensions - rather than their detail, and their impact on framework set-up - as measured by (the significance of) proposed changes. More detail is provided in Postema (2019) and in ongoing work.

\subsection{Maintaining the framework - approach}

The new modelling framework builds on the framework proposed by Monks et al. (2017) that refines the modelling framework proposed by Robinson (2008b), addressing the general class of operations systems, see Table 1 (Columns "Activity", "Detail"). In line with Robinson (2008b) Monks et al. (2017), distinguish between a number of key activities for specifying the conceptual model, see Table 1 (Column "Activity"). Each key activity is further decomposed in detailed activities. In addition, support in executing activities is offered by hinting at (i) good practices in executing them, (ii) (libraries or lists of) common choices made with respect to modelling objectives, model inputs, outputs and content (model components, attributes and their relationships) that appeal to the domain of interest, i.e. HASPs. 
Table 1 Framework extensions relative to the framework of Monks et al. (2017)

\begin{tabular}{|c|c|c|}
\hline Activity & Detail & Extensions \\
\hline $\begin{array}{l}\text { 1. Understanding } \\
\text { the problem } \\
\text { situation }\end{array}$ & $\begin{array}{l}\text { - Determine study population: Decide subcategories of the stroke } \\
\text { population the study might focus on. } \\
\text { - Assess current performance of the HASP: Interpret findings with } \\
\text { respect to their relevance for setting the modelling objectives } \\
\text { (2). } \\
\text { - Map the current process: Create a starting point for } \\
\text { determining model content (3) by building a process map that } \\
\text { captures the status quo. } \\
\text { - Explore decision variables for use in experimentation: Process } \\
\text { mapping and the (initial) assessment of HASP's current } \\
\text { performance serve as a vehicle to elicit hypotheses about delays } \\
\text { and barriers to treatment. These hypotheses are candidate } \\
\text { decision variables in model experimentation. Choice of decision } \\
\text { variables may be linked to four areas(examples): } \\
\text { O Pre-hospital logistics } \\
\text { o Processes for identification of stroke patients in the ED } \\
\text { O Communication between hospital departments } \\
\text { o Work force scheduling }\end{array}$ & $\begin{array}{l}\text { - Distinguish between LVO } \\
\text { and non-LVO patients. } \\
\text { - Establish key figures } \\
\text { concerning speed of IAT } \\
\text { treatment } \\
\text { - Process maps may span } \\
\text { both PSCs and CSCs } \\
\text { - New areas to consider are: } \\
\text { o Network topology: } \\
\text { distribution of IAT } \\
\text { services. } \\
\text { - Communication between } \\
\text { PSCs and CSCs. } \\
\text { OTransports between PSCS } \\
\text { and CSCs (patient or } \\
\text { doctor transfers). } \\
\text { Pre-hospital routing } \\
\text { decisions. }\end{array}$ \\
\hline $\begin{array}{l}\text { 2.Setting the } \\
\text { modelling } \\
\text { objectives }\end{array}$ & $\begin{array}{l}\text { - Select response measures and target performance levels: } \\
\text { o Health outcomes (primary level): The logical and time-based } \\
\text { results from a DES model can be used as input parameters to } \\
\text { either clinical models of population benefit or a health } \\
\text { economic model. } \\
\text { o Logistic performance (secondary level): Treatment volume } \\
\text { might be presented as an average rate or as a histogram of } \\
\text { the likely range of treatment rates. } \\
\text { o Target levels: Target performance levels may be set on logistic } \\
\text { performance such as treatment rates and OTT, possibly using } \\
\text { available benchmarks. } \\
\text { - Determine model outputs, see response measures and target } \\
\text { performance levels; include activity durations to assess cause } \\
\text { and effect. } \\
\text { - Determine model inputs that underlie experiments concerning } \\
\text { alternative configurations of the HASP: Construct experiments } \\
\text { that link one or more inputs to decision variables, see I. } \\
\text { - Establish restrictions in solution finding: Consider the way } \\
\text { budgets of care providers, physical space, and regulations etc. } \\
\text { may restrict choice of configurations of the stroke pathway }\end{array}$ & $\begin{array}{l}\text { - Add activity durations } \\
\text { related to patient transfer } \\
\text { and IAT treatment. } \\
\text { - See I decision variables } \\
\text { (various possible inputs for } \\
\text { areas - linking to choice of } \\
\text { organizational model) }\end{array}$ \\
\hline $\begin{array}{l}\text { 3. Determining } \\
\text { model } \\
\text { content }\end{array}$ & $\begin{array}{l}\text { - Establish model scope: Identify model boundaries, by either } \\
\text { including or excluding a representation of elements of the HASP } \\
\text { under study as model components. Choice of model } \\
\text { components is facilitated by a library of most common } \\
\text { components of a HASP, see Monks et al. (2017). } \\
\text { - Determine model detail, specifying attributes of model } \\
\text { components. See Monks et al. (2017) for entity attributes that } \\
\text { may be required across a broad range of objectives. } \\
\text { - Specify assumptions underlying model content: Facilitate the } \\
\text { interpretation of the model and its workings by making } \\
\text { assumptions on the HASP under study explicit. See Monks et al. } \\
\text { (2017) for common assumptions on HASP simulation models. } \\
\text { Making appropriate model simplifications: For common } \\
\text { simplifications of HASP simulation models see Monks et al. } \\
\text { (2017). }\end{array}$ & $\begin{array}{l}\text { - Add model components } \\
\text { representing intra hospital } \\
\text { activities associated with } \\
\text { IAT treatment, inter } \\
\text { hospital patient and doctor } \\
\text { transfer, and on-scene } \\
\text { patient screening for LVO } \\
\text { to library of components of } \\
\text { a HASP (see Figure 1). } \\
\text { - Include LVO/Non-LVO in } \\
\text { patient classification. }\end{array}$ \\
\hline
\end{tabular}


Framework development relies on five sources of information: (i) literature reporting on simulation studies concerning IVT - compare Monks et al. (2017), (ii) literature reviews on alternative organization models for IAT (Dètraz et al., 2018, Ciccone et al., 2019), (iii) references as in (ii), (iv) interviews with domain experts and (v) authors' involvement in stroke research, including doing simulation studies. Initial validation of the framework is done by domain experts. Note that, so far, simulation studies concerning organization models for IAT are envisioned, but not reported in literature.

\subsection{Framework extensions}

Implementation of IAT treatment sets new requirements to HASP setup. In turn, these requirements are to be reflected in simulation study setup, in terms of modelling objectives, model inputs, outputs and content (Table 1 (Column "Extensions")).

Essentially, decision making on HASP setup may be considered at three levels, i.e., strategic, tactical and operational, thereby acknowledging the time horizon at which changes may be implemented, their impact on patient outcomes, and their associated costs and efforts. Choices with respect to the network topology, i.e., the distribution of stroke service over the region, are considered strategic decisions. IAT services are typically distributed over a restricted number of hospitals, due to the low number of patients involved, requirements set on expertise and (staff) resources, and natural and organic growth of stroke services already located and distributed in certain regions. The tactical level considers patient routing along stroke services, compare the DS and MS models. Finally, operational level decisions involve expediting care and transport services along the pathway. Decision variables related to each level, candidating as model inputs, are shown in Table 1 (Column "Extensions"), see activities 1 and 2.

Current clinical practice is dominated by the DS and MS organizational models. Taken together they organize regional stroke care. Both models involve system elements and activities that are new relative to the "classic" IVT-only based organization models. Newly proposed interventions (see Section 2) bring further elements and detail. Elements identified are to be reflected in new types of model components and their attributes for specifying model content, see Table 1 (Column "Extensions"), activity 3. In turn, new methods or rules for model simplification, or common assumptions, may guide component use and choice of their detail, in order to enhance model utility and feasibility.

Choice of model outputs is influenced by the new spectrum of system elements, and associated decision variables - linking to those elements open for change. To assess effects of new model inputs on system performance, further detailed outputs are required, especially for capturing delays associated with new care and transport services.

\section{DISCUSSION - MODELLING FRAMEWORKS AS ASSETS}

\subsection{The need for maintenance}

We observed a need for modifications of a domain specific modelling framework for HASP simulation, given the availability of new treatments, and innovations foreseen in service delivery, having significant impact on organizing HASPs. Such system changes are likely to occur, also in other domains. Hence, domain specific modelling frameworks, being tailored towards system specifics for a domain, cannot do without maintenance, in order to keep up their service levels. So far, the observed need for maintenance has not been acknowledged in literature.

\subsection{What has been done?}

Starting from several information sources, including literature, interviews with domain experts and authors' involvement in stroke research, including simulation studies, modelling framework extensions are developed. Extensions concerned (libraries or lists of) common choices made with respect to modelling objectives, model inputs, outputs and content (model components, attributes and 
their relationships) that appeal to the domain of interest, i.e. HASPs. Essentially, changes, despite being significant, do not address the framework core, i.e., key modelling activities.

\subsection{When to update?}

The need to do maintenance was fostered by the authors' wishes of doing HASP simulation with IAT in the near future. While the framework serves their purposes, one may wonder about the timing of the initiative. At the decision moment no evidence of simulation studies with IAT was available. Furthermore, significant learning effects are observed in clinical practice in mastering the new DS and MS organization models, while new organization models are introduced at high pace.

\subsection{Investment made}

Efforts made, i.e., several months of full-time work, do classify modelling framework extensions as major maintenance. Much time is invested by one of the authors in familiarizing himself with the original framework - not being one of its developers, and not being involved in the domain before. Increased complexity and uncertainty associated with organization models for IAT further add to this, also see above (When to update?). Findings suggest how framework maintenance may boil down to a significant investment, requiring a clear project definition and tailored team - being familiar with the domain.

\subsection{Design for maintenance}

The observed need for modelling framework maintenance is clear. However, the way domain-based changes are to be related to the framework is somewhat less clear. Which elements have to be considered for possible modification? This raises issues like standards for framework set-up, and the modularity of their setup.

\subsection{Maintenance policies - what and how to respond to?}

Our case study considers an incident - an observed need for major maintenance of domain specific elements of the modelling framework due to significant changes of referent systems. What are possible other needs: improved modelling methodology, new methods, new best practices, ....? How to respond to this? Should we link this to timewise or status dependent triggers? The issue is relevant for simulation users in industry and education.

\subsection{Limitations}

Our findings are based on a single case study. Clearly, further studies are required to explore issues raised in greater depth. However, the study clarifies how the issue is very much there - in practice where modelling frameworks whether explicit or implicit are the practitioner's or student's assets that are in need of regular maintenance.

\section{CONCLUDING REMARKS}

In this article we explore the needs for maintenance of simulation modelling frameworks, requirements maintenance imposes on framework set-up, structure of maintenance policies and maintenance process. Starting from a case study on a new modelling framework for HASP simulation we establish a general need for maintenance of modelling frameworks - to benefit educators, students and simulation practitioners.

Case findings suggest how maintenance may take considerable efforts of developers in familiarizing with the original framework, the domain and its new facets. Therefore, policies are required clarifying what, when and how to maintain. Tailoring framework design towards its maintenance is considered instrumental for effective and efficient maintenance.

Future research is directed towards developing modelling frameworks for HASPs, including nonischemic stroke patients, framework maintenance and framework modularity and standards. A starting point may be found in related research on software maintenance and quality (Malhotra and Chug, 2016; Stevenson and Wood, 2018). 


\section{REFERENCES}

Arbez G and Birta L (2011). The ABCmod Conceptual Modelling Framework. In: Robinson S, Brooks R J, Kotiadis K. and Van der Zee D J (eds). Conceptual Modelling for Discrete-Event Simulation. CRC/Taylor \& Francis: Boca Raton, pp 133-178.

Berkhemer O A, Fransen P S, Beumer, D et al. (2015). A Randomized Trial of Intraarterial Treatment for Acute Ischemic Stroke. New England Journal of Medicine 372: 11-20.

Chia NH , Leyden J M, Newbury J, et al. (2016). Determining the Number of Ischemic Strokes Potentially Eligible for Endovascular Thrombectomy: A Population-Based Study. Stroke 47:13771380.

Ciccone, A, Berge, E and Fischer, U (2019). Systematic review of organizational models for intraarterial treatment of acute ischemic stroke. International Journal of Stroke 14(1): 12-22.

Détraz, L, Ernst, M and Bourcier, R (2018). Stroke Transfer and its Organizational Paradigm Review of Organizational Paradigms and the Impact on Outcome. Clinical Neuroradiology 28: 473-480.

Emberson J, Lees K R, Lyden P, et al. (2014). Effect of treatment delay, age, and stroke severity on the effects of intravenous thrombolysis with alteplase for acute ischaemic stroke: a meta-analysis of individual patient data from randomised trials. Lancet 384(9958): 1929-1935.

Furian, N, O'Sullivan, M, Walker, C, Vössner, S and Neubacher, D (2015). A conceptual modeling framework for discrete event simulation using hierarchical control structures. Simulation Modelling Practice \& Theory 56: 82-96.

Karagoz N A and Demirors O (2010). Conceptual Modelling Notations and Techniques. In: Robinson S, Brooks R J, Kotiadis K. and Van der Zee D J (eds). Conceptual Modelling for Discrete-Event Simulation. CRC/Taylor \& Francis: Boca Raton, pp 179-210.

Kotiadis K, Tako A and Vasilakis C (2014). A participative and facilitative conceptual modelling framework for discrete event simulation studies in healthcare. Journal of the Operational Research Society 65(2): 197-213.

Law, A M (1991). Simulation Model's Level of Detail Determines Effectiveness. Industrial Engineering, 23 (10): 16-18.

Malhotra, R and Chug, A (2016) Software Maintainability: Systematic Literature Review and Current Trends. International Journal of software engineering and knowledge engineering 26(8): 12211253.

Monks, T, van der Zee, D J, Lahr, M, Allen, M, Pearn, K, James, M A, Buskens, E and Luijckx, G J (2017). A framework to accelerate simulation studies of hyperacute stroke systems. Operations Research for Health Care 15: 57-67.

Pace D K (1999). Development and documentation of a simulation conceptual model. In Proceedings of the 1999 Fall Simulation Interoperability Workshop. Available via www.sisostds.org.

Pace D K (2000). Simulation conceptual model development. In Proceedings of the 2000 Spring Simulation Interoperability Workshop. Available via www.sisostds.org.

Postema, F H (2019). A modeling framework for hyperacute stroke care simulation with endovascular treatment. MSc thesis, University of Groningen.

Robinson S (2008a). Conceptual modelling for simulation Part I: definition and requirements. Journal of the Operational Research Society 59(3): 278-290.

Robinson S (2008b). Conceptual modelling for simulation Part II: a framework for conceptual modelling. Journal of the Operational Research Society 59(3): 291-304.

Robinson S (2019). Conceptual modelling for simulation: Progress and grand challenges. Journal of Simulation (in press).

Saver J L, Goyal M, van der Lugt A, et al. (2016). Time to Treatment With Endovascular Thrombectomy and Outcomes From Ischemic Stroke: A Meta-analysis. JAMA 316(12): 1279.

Stevenson, J and Wood, M (2018) Recognising object-oriented software design quality: a practitionerbased questionnaire survey. Software Quality Journal 26(2): 321-365.

The National Institute of Neurological Disorders and Stroke rt-PA Stroke Study Group (1995). Tissue plasminogen activator for acute ischemic stroke. New England Journal of Medicine 333:15811587.

van der Zee, D J (2019). Model Simplification in Manufacturing Simulation - Review and Framework. Computers \& Industrial Engineering: 127: 1056-1067. 
Van der Zee D J and Van der Vorst J G A J. (2005). A modeling framework for supply chain simulation - Opportunities for improved decision-making. Decision Sciences 36(1): 65-95.

Van der Zee D J, Brooks R J, Robinson S and Kotiadis K (2011). Conceptual Modelling: Past, Present and Future. In: Robinson S, Brooks R J, Kotiadis K. and Van der Zee D J (eds). Conceptual Modelling for Discrete-Event Simulation. CRC/Taylor \& Francis: Boca Raton, pp 473-490.

\section{AUTHOR BIOGRAPHIES}

DURK-JOUKE VAN DER ZEE is associate professor of Operations at the Faculty of Economics and Business, University of Groningen, The Netherlands. He holds a MSc and Phd in Industrial Engineering. His research interests include health care logistics engineering, simulation methodology and applications, simulation \& serious gaming, and manufacturing planning \& control. He is a member of the INFORMS-SIM. His webpage is http://www.rug.nl/staff/d.j.van.der.zee.

FRISO POSTEMA is a junior researcher at the Faculty of Economics and Business, University of Groningen, The Netherlands. Currently he is working as a junior supply chain analyst in the private sector. His research interests include simulation methodology and applications in health care logistics.

THOMAS MONKS is an Associate Professor of Health Data Science at the University of Exeter Medical School and The Institute of Data Science and AI, University of Exeter. He is also Turing Fellow at The Alan Turing Institute and Honorary researcher at the Clinical Operational Research Unit, UCL. He holds a BSc in Computer Science and Mathematics, MSc in Operational Research and $\mathrm{PhD}$ in Simulation Modelling. Between 2014 and 2019 he led an applied healthcare Data Science team funded by the NIHR. He has worked as both a Software Engineer in the private sector and an Operational Research Analyst within the public sector. His speciality is simulation of healthcare systems of unscheduled and emergency care. He was co-chair of the UKs Simulation Workshop 2016 and 2018. His webpage is: https://www.turing.ac.uk/people/researchers/thomas-monks

WILLEMIJN J. MAAS is a PhD Candidate at the Department of Neurology and the HTA unit of the Department of Epidemiology, University of Groningen, University Medical Center Groningen, The Netherlands. Her research interests include the organization of acute stroke care and discrete-event simulation.

MAARTEN M. H. LAHR is a postdoctoral researcher at the HTA unit of the Department of Epidemiology, and member of the Healthy Ageing Team, University of Groningen, University Medical Center Groningen, The Netherlands. His research interests include organizational models for acute stroke care and discrete-event simulation. His website is http://www.rug.nl/staff/m.m.h.lahr/.

MAARTEN UYTTENBOOGAART is an interventional neurologist at the department of Neurology and Radiology, University Medical Center Groningen. He is involved in the Cerebrovascular Unit, and Principal Investigator at the Research School of Behavioural and Cognitive Neurosciences Groningen (BCN) and KOLFF institute. His research topics are clinical research on prognostic factors in and treatments for acute ischemic stroke, clinical research on neuroprotection in acute stroke and research on organization and implementation of acute stroke care.

ERIK BUSKENS is Professor of Health Technology Assessment University Medical Center Groningen, and faculty of Economics and Business University of Groningen, The Netherlands. His research interests among others encompass the association and mutual dependencies between technological advances and the factual organization of care to attain the desired health and possibly economic benefits. 